\title{
Lateral Non-covalent Clamping of Graphene at the Edges Using a Lipid Scaffold
}

\author{
Lia M. C. Lima, Hadi Arjmandi-Tash, and Grégory F. Schneider* \\ Faculty of Science, Leiden Institute of Chemistry, Leiden University, Einsteinweg 55, 2333CC Leiden, The Netherlands
}

Supporting Information

ABSTRACT: Developing a clean handling and transfer process, capable of preserving the integrity of two-dimensional materials, is still a challenge. Here, we present a flexible, dynamic, and lipid-based scaffold that clamps graphene at the edges providing a practical, simple, and clean graphene manipulation and transfer method. Lipid films with different surface pressures are deposited at the air/copperetchant interface immediately after placing the graphene samples. We show that at surface pressures above $30 \mathrm{mN} / \mathrm{m}$, the lateral support prevents graphene movement and cracking during all etching and transfer. The method provides new insights into the handling of graphene and can yield efficient, sensitive, and clean graphene-based devices.

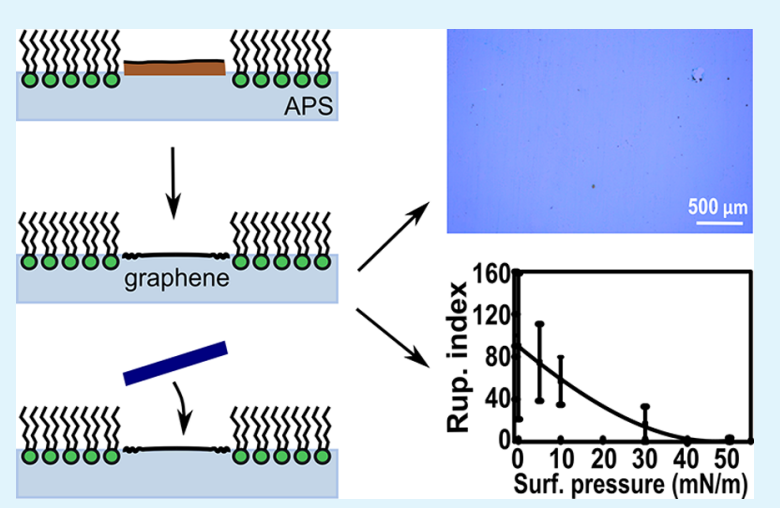

KEYWORDS: lipid scaffold, graphene, Langmuir-Blodgett trough, Langmuir films, graphene polymer-free transfer

\section{INTRODUCTION}

Over the last years, a growing interest in graphene led to the development of novel sensing devices. ${ }^{1-5}$ However, for the realization of such devices, graphene has to pass through several fabrication steps. $^{6-9}$ Large-scale graphene sheets are typically synthesized on metallic catalysts ${ }^{10}$ and then transferred to target substrates such as $\mathrm{Si} / \mathrm{SiO}_{2}$ wafers. ${ }^{11,12}$ The transfer process can highly degrade the properties of graphene and its performance in the final applications. ${ }^{13}$ The immobilization of two-dimensional (2D) materials (e.g., graphene) by Langmuir films is a strategy to controllably manipulate graphene directly in an aqueous environment, without using polymeric scaffolds. ${ }^{14}$ Langmuir films of amphiphilic lipid molecules are insoluble at the air/liquid interface generating a lateral pressure profile due to the intermolecular forces in the lipid monolayer. The film is stable and $2 \mathrm{D}$, guaranteeing a strictly lateral compression (no perpendicular component which may cause out-of-plane deformation) of graphene. The molecules act to decrease the surface tension of the subphase, therefore creating a dynamic clamp which adjusts itself according to the geometry of graphene. For the first time, lipids are introduced as molecular springs clamping graphene from the sides at the air/ liquid interface. The flexible lipid-based scaffold prevents graphene movement and cracking during copper etching and later on, during transfer onto the target substrate. We investigate the performance of the lipid clamp and compare it with existing polymer-free transfer methods. ${ }^{16-18}$

In a Langmuir-Blodgett (LB) trough ${ }^{14}$ the lipids are deposited at the air/liquid interface and compressed passing through different characteristic phases, namely, the gaseous (G) state, the liquid expanded (LE) state, the liquid condensed
(LC) state, and the solid (S) state (Figure 1a). ${ }^{19-21}$ In the presence of graphene, as the intermolecular distance between the lipid molecules decreases during compression, the force exerted by both the hydrophobic acyl chains and the hydrophilic polar groups induces a pressure on graphene edges; the increase in the surface pressure $(\pi)$ is directly linked to how closely the lipids are packed. Therefore, the dynamic pressure of the lipid monolayer can keep the domains of graphene together and intact, preventing the growth of already existing cracks (e.g., through grain boundaries). ${ }^{22}$

The most common graphene transfer method includes a temporarily coating of graphene with a polymeric film while etching the metal substrate with an etchant solution. The coating acts as a mechanical support and prevents graphene cracking. The coating is thereafter removed by suitable chemicals once graphene is transferred onto the target substrate. Leftovers of polymer residuals are the important limitation of the technique, which may degrade the ultimate quality of graphene. ${ }^{23-26}$ Therefore, in the recent years, novel polymer-free transfer methods have been developed, most of which use physical supports such as graphite holders ${ }^{16}$ or transmission electron microscopy grids, ${ }^{17}$ limiting the flexibility of the process. Alternatively, graphene is also covered with organic solvents such as cyclohexane ${ }^{9}$ or hexane, ${ }^{18}$ avoiding polymer contaminations.

In our method, as the surface of graphene is not in contact with any physical support, the basal plane remains uncon-

Received: January 17, 2018

Accepted: March 7, 2018

Published: March 7, 2018 

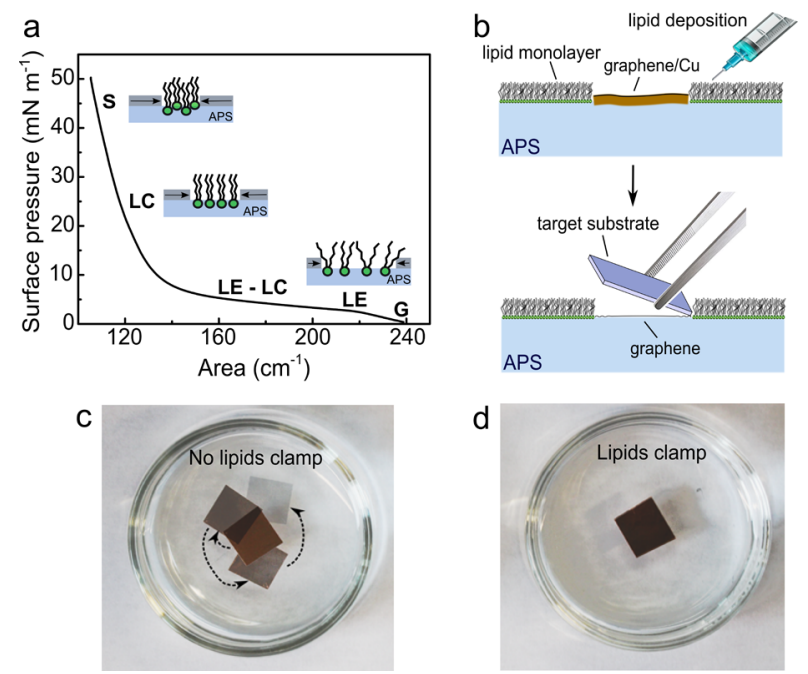

Figure 1. (a) Surface pressure-area compression isotherm of 1,2dipalmitoyl-sn-glycero-3-phosphocholine (DPPC) lipid monolayer on ammonium persulfate aqueous solution (APS) at $25{ }^{\circ} \mathrm{C}$ showing different phase separations. G: gaseous state; LE: liquid expanded state; LC: liquid condensed state; and S: solid state. (b) Illustration of the molecular edge clamp concept. (c,d) Time-lapse photographs of a piece of graphene grown on copper floating on a solution of $0.5 \mathrm{M}$ APS in water without (c) and with (d) the lipid clamp.

taminated, allowing the realization of clean devices directly on water. With this non-covalent bonding, graphene can be manipulated from the edges, leading additionally to an optimal transfer to arbitrary substrates. Increasing the lateral pressure of the lipids decreases the number of cracks and further preserves the quality of the transfer. The development of a lateral support damps vibrations on the surface of the etchant (happening during etching or transfer), which may induce cracks. This immobilization by Langmuir films establishes a novel strategy for fundamental studies of graphene and also for transfer purposes. For different applications, ${ }^{15,27,28}$ particularly to realize electronic devices, our introduced method could solve contamination issues; furthermore, our novel platform serves to manipulate and study graphene on a liquid support.

\section{EXPERIMENTAL SECTION}

LB Technique. 1,2-Dipalmitoyl-sn-glycero-3-phosphocholine (DPPC) lipids (Avanti Polar Lipids Inc.) with a concentration of 1 $\mathrm{mg} / \mathrm{mL}$ were dissolved in $\mathrm{CHCl}_{3} / \mathrm{CH}_{3} \mathrm{OH}$ 3:1 vol \%. The lipid solution was deposited dropwise on an ammonium persulfate solution (APS, $0.5 \mathrm{M}$ ) at $25{ }^{\circ} \mathrm{C}$ using a microliter syringe, and the solvent was allowed to evaporate for $15 \mathrm{~min}$. The measurements were performed in a Minitrough 2, KSV Instruments, using KSV Research Lab v2.01 software. The teflon trough of the setup was equipped with hydrophilic barriers made of Delrin.

Graphene Transfer. Six Petri dishes filled with APS were prepared prior to the experiments. Copper foils with chemically grown graphene (all with an approximate size of $\sim 10 \times 10 \mathrm{~mm}^{2}$ ) were placed on the surface of the etchant (Figure S1, Supporting Information). Immediately afterwards, the DPPC lipids were added to the air/ etchant interface with different $\pi(0-50 \mathrm{mN} / \mathrm{m})$ in separate Petri dishes. After the copper etching, the graphene floating at the interface was transferred to different $\mathrm{Si} / \mathrm{SiO}_{2}$ substrates by gently putting the substrate in contact with graphene. Eventually, APS residues remaining on the surface of graphene were rinsed with a continuous flow of ultrapure water. The $\pi<10 \mathrm{mN} / \mathrm{m}$ is insufficient to prevent the lateral movements of the graphene or preserve its integrity during etching (see the graphene position on Figure S1a,b). Instead, for $\pi \geq 30 \mathrm{mN}$ / $\mathrm{m}$, the foil (graphene) remained immobilized. Higher $\pi$ fixes the foil in position throughout the etching. However, at the $\pi$ of $40 \mathrm{mN} / \mathrm{m}$, the graphene sheet was slightly contracted because of the force induced by the lipids on the graphene edges. Importantly, we did not observe any remarkable contraction of graphene at $50 \mathrm{mN} / \mathrm{m}$, which suggests that the lipids collapsed ${ }^{29}$ (i.e., overlapped or sank), reducing the overall $\pi$.

An alternative transfer approach was to place the $\mathrm{Si} / \mathrm{SiO}_{2}$ substrate at the bottom of the Petri dish. After the copper etching, the APS was replaced by ultrapure water with a continuous flow of water in and APS out. Eventually, the surface of the water was lowered to gently place graphene onto the substrate. This approach also achieves the transfer of a continuous graphene, as observed by the optical micrograph (Figure S2, Supporting Information), confirming the versatility of the molecular edge clamp concept.

Characterization. Imaging. Optical images were obtained by a Leica DM 2700 M microscope with a $5 \times$ objective. Fluorescence quenching microscopy for quantifying the amount of the cracks in graphene was performed with a $20 \times$ objective on a Axiovert 200 ZEISS inverted fluorescence microscope equipped with a monochrome AxioCam MRm ZEISS camera. To do this measurement, the surface of graphene was spin-coated with a poly(methyl methacrylate) (PMMA) layer premixed with $2-6 \mu \mathrm{L}$ solution of rhodamine $\mathrm{B}, 4 \mathrm{mM}$ (dissolved in acetone). The captured images were postprocessed according to the protocol we published earlier ${ }^{30}$ to estimate the rupture index $(\mathrm{RI})$.

Infrared External Reflection Spectroscopy. Spectra were collected with a Bio-Rad Excalibur Series infrared spectrometer in external reflectance mode. The chamber was continuously purged with dry air, and a sample of bare $\mathrm{Si} / \mathrm{SiO}_{2}$ substrate was taken as the reference. The angle of incidence was measured at $45^{\circ}$ with a spectra range of 8.000$0 \mathrm{~cm}^{-1}$. Each spectrum was collected for an average of 128 scans with a resolution of $4 \mathrm{~cm}^{-1}$

Raman Spectroscopy. Raman spectra were measured on a WiTec alpha 3000 confocal spectrometer with a $532 \mathrm{~nm}$ laser excitation source and a $100 \times$ objective. Each sample was measured at least on three different locations, to obtain an average spectrum.

Atomic Force Microscopy. Atomic force microscopy (AFM) images were obtained with a JPK NanoWizard Ultra Speed instrument, and the images were processed on JPK SPM Data Processing software. The experiments were performed using a silicon probe (AC240TS, Asylum Research) with $300 \mathrm{kHz}$ nominal resonance frequency. The images were scanned in an intermittent contact mode at room temperature with $512 \times 512$ pixels.

\section{RESULTS AND DISCUSSION}

Molecular Edge Clamp Preparation. The LB trough is an efficient apparatus to form well-packed lipid monolayers at the air/liquid interface. The DPPC monolayer was compressed up to $\pi=50 \mathrm{mN} / \mathrm{m}$ (Figure 1a). The recorded isotherms were used to reproduce the same lipid $\pi /$ area conditions in six different Petri dishes (see Experimental Section and Supporting Information). All experiments were carried out at room temperature $\left(\sim 25^{\circ} \mathrm{C}\right)$ where DPPC is in the gel phase. Note that DPPC has a main phase transition temperature $\left(T_{\mathrm{m}}\right)$ of 41 ${ }^{\circ} \mathrm{C}$. Below the $T_{\mathrm{m}}$, the DPPC monolayer presents different phases during compression, as observed in Figure 1a. Figure $1 \mathrm{~b}$ illustrates the concept of the molecular edge clamp. A sufficiently high $\pi$ prevents any noticeable movement of graphene during all etching procedures and forms a wellordered and compact layer that holds the graphene in place through all processes. Figure $1 \mathrm{c}, \mathrm{d}$ shows overlapped snapshots at different copper etching stages of two samples with and without the lipid clamp at the edges. Clearly, the graphene without any lateral support moves randomly, which may eventually promote its cracking. Instead, lipids on the surroundings of graphene hold the graphene in place during all etching and transfer, preserving its integrity (see video, Supporting Information). 
Integrity of Transferred Graphene. Figure 2 compares the optical images of graphene samples transferred onto $\mathrm{Si}$ /

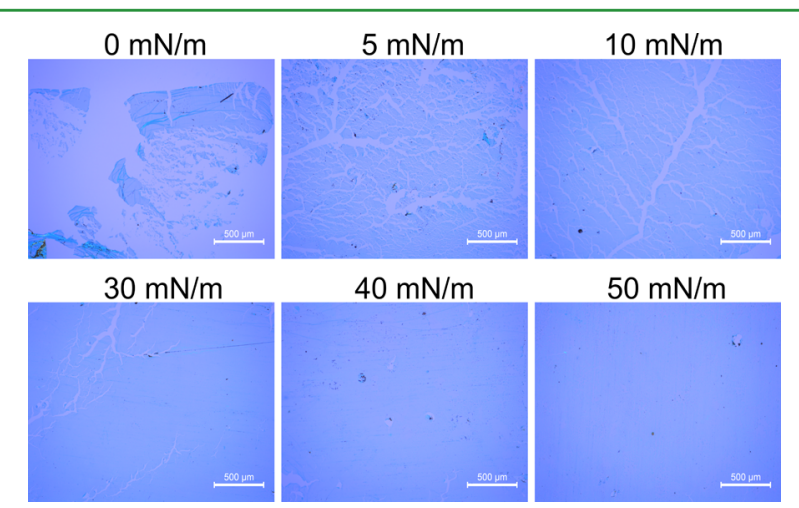

Figure 2. Optical micrographs of graphene on $\mathrm{Si} / \mathrm{SiO}_{2}$ substrates transferred at different clamp pressures $(0,5,10,30,40$ and $50 \mathrm{mN}$ / m). Scale bars are $500 \mu \mathrm{m}$.

$\mathrm{SiO}_{2}$ substrates with different lateral pressures. In fact, the sample without any lipid lateral support is unsteady and dramatically loses its integrity. The low-amplitude vibrations of the surface of the etchant and the transfer could be responsible for such damages. The graphene sheets transferred in the presence of a strong lipid edge clamp, however, are continuous to a large extent: increasing the $\pi$ prevents the formation of cracks, showing the advantage of the lateral clamp support. Particularly, at the LC state $(\pi>30 \mathrm{mN} / \mathrm{m})$, the interaction between the lipid molecules at the interface is large enough to induce a high pressure on the edges of graphene and promotes a stable clamp while transferring to the $\mathrm{Si} / \mathrm{SiO}_{2}$ substrates.

$\mathrm{RI}$ provides a quantitative measure of the integrity of graphene samples. ${ }^{30}$ The emission of the fluorophore molecules in close vicinity to graphene is quenched via a peculiar energytransfer mechanism, leading to an outstanding contrast between cracked and continuous graphene areas in fluorescence microscopy (see the images in the top row in Figure 3). RI is determined by counting the number of pixels localized at the border of the cracks (white pixels visible in the bottom row of Figure 3) and divided by the total number of pixels corresponding to graphene multiplied by one thousand. In application, the median of different RI values measured at several spots on the sample is reported to exclude the effect of any local inhomogeneities (see Figure 4). Remarkably, increasing the $\pi$ using the lipid edge clamp has a direct effect on the integrity of graphene. Particularly, $\pi \geq 40 \mathrm{mN} / \mathrm{m}$ provides a negligible RI, comparable to graphene transferred with a PMMA coating. The RI results are in line with the optical microscopy studies. At high $\pi$, the lipid monolayer becomes more compact with smaller intermolecular areas reflecting on a more confined lipid clamp, therefore preserving the original state of graphene upon transfer to the $\mathrm{Si} / \mathrm{SiO}_{2}$ substrate.

The $\pi$ affects the duration of the copper foil etching $\left(t_{\text {etch }}\right)$. Figure 5 a plots the estimated $t_{\text {etch }}$ as the function of $\pi$ : we use the time span between the moment some parts of the foil started to be transparent up to the moment the foil turns to be fully invisible to define the etching time. Generally, increasing the $\pi$ increases $t_{\text {etch }}$. Indeed, the very high intermolecular interactions between lipid molecules most probably drive lipids toward the foil/etchant interface resulting in delays of the etching as migrated lipids may form a layer between the copper

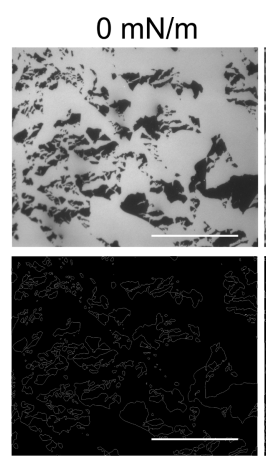

$30 \mathrm{mN} / \mathrm{m}$
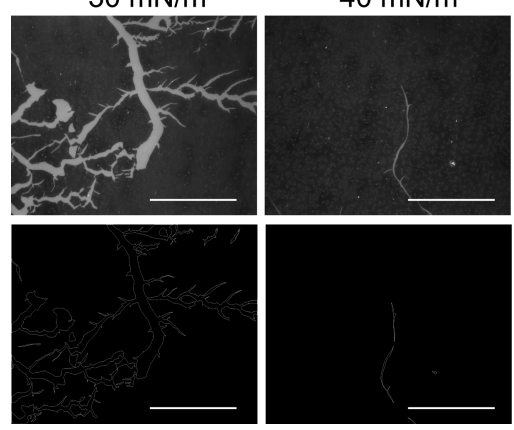

$5 \mathrm{mN} / \mathrm{m}$

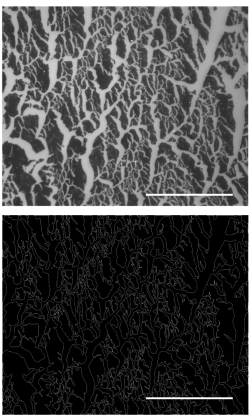

$40 \mathrm{mN} / \mathrm{m}$

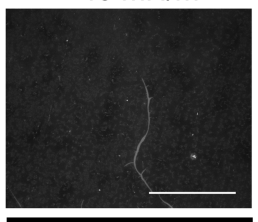

$50 \mathrm{mN} / \mathrm{m}$
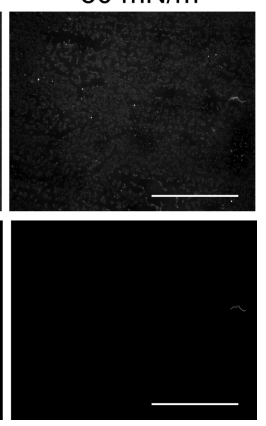

Figure 3. Fluorescence quenching microscopy images of graphene on $\mathrm{Si} / \mathrm{SiO}_{2}$ substrates with different surface pressures of the lipids (top row) and the corresponding images after processing (bottom row). All scale bars correspond to $50 \mu \mathrm{m}$.

and the etchant. The delay depends on the concentration of the intercalated lipid molecules, the latter being proportional to the $\pi$. At the highest $\pi(50 \mathrm{mN} / \mathrm{m})$, presumably a considerable amount of the lipids collapsed (reducing the effective $\pi$ ), in line with what is observed by Figure S1, Supporting Information.

Characterization of Transferred Graphene. Infrared external reflection spectroscopy (IR-ERS) measurements can probe the lipid/graphene interactions after the transfer onto $\mathrm{Si} /$ $\mathrm{SiO}_{2}$ substrates. As seen from Figure $5 b$, no significant absorption bands characteristic for the stretching vibrations of the lipid acyl chains were detected. The lack of lipid traces suggests that during the transfer, the affinity between the lipid molecules intercalated underneath graphene and the etchant is stronger than the lipid-graphene interaction, allowing the molecules to remain at the interface. Alternatively, the step of rinsing with ultrapure water after the transfer of graphene-to remove the remaining etchant residues - could have rinsed the lipids away. For the purpose of comparison, we measured the infrared spectra of a well-organized lipid monolayer transferred onto a $\mathrm{Si} / \mathrm{SiO}_{2}$ substrate using $\mathrm{LB}$ trough at $\pi=30 \mathrm{mN} / \mathrm{m}$ with a graphene sheet above (Figure $5 b$, orange line-LB DPPC). ${ }^{31}$ The results show an intense absorbance on the symmetric $\left(\sim 2844 \mathrm{~cm}^{-1}\right)$ and asymmetric $\left(\sim 2912 \mathrm{~cm}^{-1}\right)$ methylene vibrations of the lipid acyl chains even for a single layer of lipids, further confirming the assumption that the lipids from the clamp were not transferred. Additionally, the black line corresponding to the graphene without any lipids at the interface is comparable to any other spectra of graphene transferred with different $\pi$, further emphasizing that the lipids were indeed not transferred with the graphene to the $\mathrm{Si} / \mathrm{SiO}_{2}$ substrates. Such observations further confirm that the molecular edge clamp provides a clean transfer to realize sensitive graphene-based devices. 

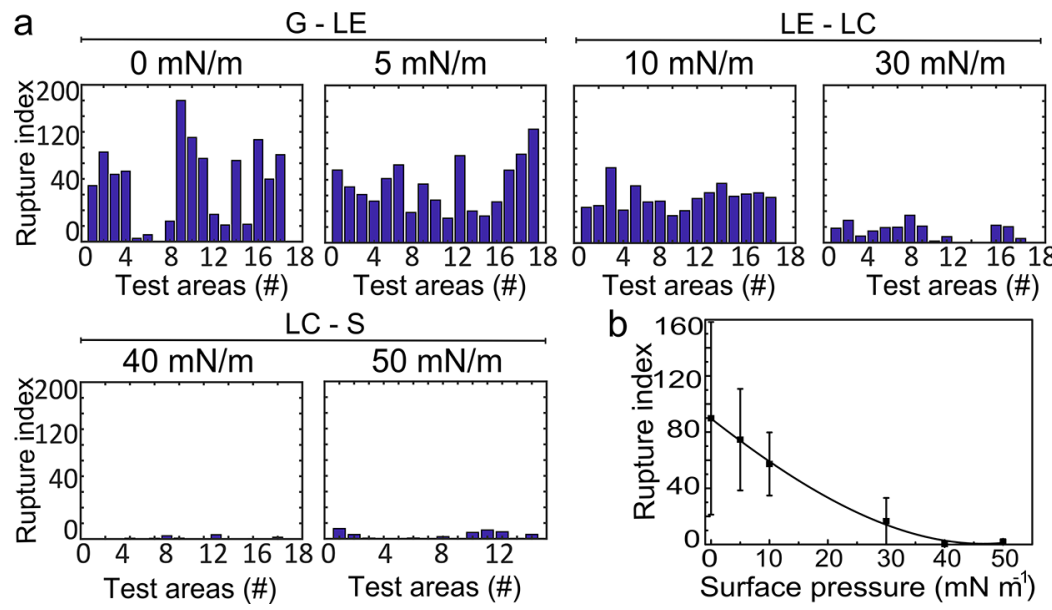

Figure 4. (a) Quantitative characterization of crack formation at different lateral clamping strengths for surface pressures ranging from 0 to $50 \mathrm{mN} /$ m.G-LE: gaseous to liquid expanded state; LE-LC: liquid expanded to liquid condensed state; and LC-S: liquid condensed to solid state. Dimensionless RI was estimated for a maximum of 18 different arbitrarily selected windows (test areas, $x$ axis) on the sample. (b) Median RI values in (a) as a function of the surface pressure; the solid line is the fit using a polynomial function.
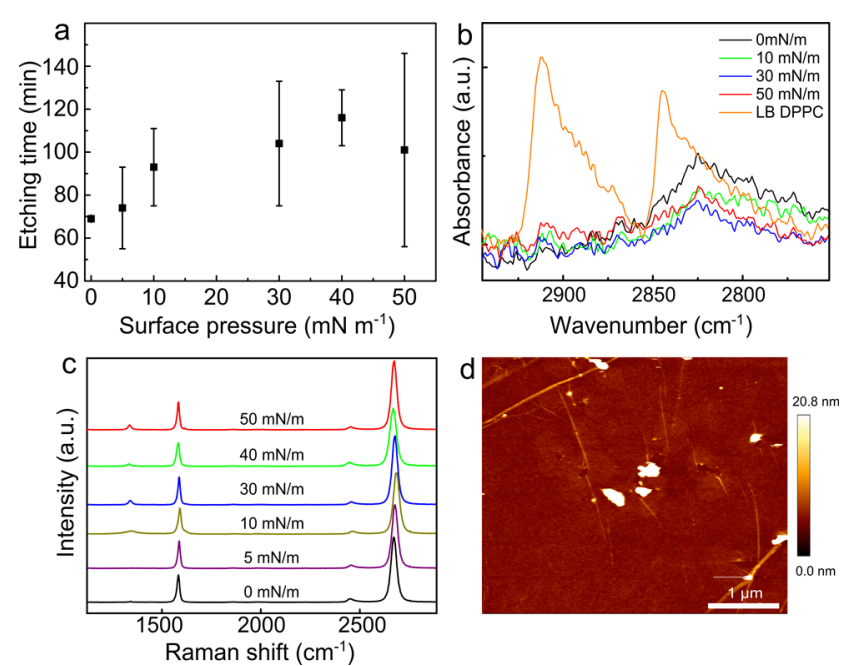

Figure 5. (a) Etching time of the copper foil as a function of the surface pressure. (b) IR-ERS spectra of graphene transferred at different surface pressures of lipids. An additional spectrum corresponding to the transfer of a DPPC lipid monolayer by LB with a graphene sheet above is also presented. (c) Raman spectra of different graphene samples on $\mathrm{Si} / \mathrm{SiO}_{2}$ substrates transferred with different surface pressures $(0,5,10,30,40$, and $50 \mathrm{mN} / \mathrm{m})$. (d) AFM intermittent contact mode image in air at room temperature of graphene transferred to an $\mathrm{Si} / \mathrm{SiO}_{2}$ substrate with a surface pressure of $30 \mathrm{mN} / \mathrm{m}$

Separately, the quality of the graphene transferred at different $\pi$ was analyzed by Raman spectroscopy after the transfer on $\mathrm{Si} /$ $\mathrm{SiO}_{2}$ substrates (Figure 5c). Remarkably, Raman spectra are insensitive to $\pi$ and feature a $2 \mathrm{D}$ peak $\left(\sim 2680 \mathrm{~cm}^{-1}\right)$ characteristic of a single-layer graphene, a weak $D$ peak at $\sim 1350 \mathrm{~cm}^{-1}$, and a sharp G peak $\left(\sim 1590 \mathrm{~cm}^{-1}\right)$, confirming the outstanding quality of graphene after transfer. Similarly, Figure 5d shows an AFM image of continuous graphene on the $\mathrm{Si} / \mathrm{SiO}_{2}$ substrate transferred at $\pi=30 \mathrm{mN} / \mathrm{m}$. In line with what was mentioned above, graphene is fully immobilized by the lipid molecules when in the LC state or above, because of the increasing intermolecular interactions in the monolayer that reduces the surface tension and creates a flexible scaffold on the edges of graphene. This prevents graphene from cracking during all etching time and consequently after the transfer to $\mathrm{Si} / \mathrm{SiO}_{2}$ substrates.

\section{CONCLUSION}

We present a molecular lipid-based scaffold that laterally clamps graphene from the edges leading to an optimal manipulation and transfer of graphene to arbitrary substrates, preventing polymeric contamination and cracking of graphene. The method is based on laterally supporting graphene with a lipid monolayer on the surface of the etchant.

We investigated the uniformity of the graphene by means of optical images and RI. The integrity of graphene increases by increasing the lipid lateral pressure. The surface of graphene is clean without process residuals. Compared to other existing polymer-free transfer methods, this process benefits from the fact that the surface of graphene is not in contact with any physical support, the process is flexible and straightforward. One immediate possible application would be to study the electrical and mechanical properties of graphene with lateral compression of the lipid scaffold.

\section{ASSOCIATED CONTENT}

\section{S Supporting Information}

The Supporting Information is available free of charge on the ACS Publications website at DOI: 10.1021/acsami.8b00916.

Clamping graphene with lipids (ZIP)

Photographs of six Petri dishes with graphene clamped with different surface pressures of lipids $(0,5,10,30,40$, and $50 \mathrm{mN} / \mathrm{m}$ ) before and after the etching of the copper and an alternative molecular edge clamp concept with the substrate on the bottom of the Petri dish with the corresponding optical image and Raman results for a lipid surface pressure of $30 \mathrm{mN} / \mathrm{m}$ (PDF)

\section{AUTHOR INFORMATION}

\section{Corresponding Author}

*E-mail: g.f.schneider@chem.leidenuniv.nl.

\section{Notes}

The authors declare no competing financial interest. 


\section{ACKNOWLEDGMENTS}

This research was gratefully funded by the European Research Council under the European Union's Seventh Framework Programme (FP/2007-2013)/ERC grant agreement no. 335879 project acronym "Biographene". We thank F. Galli for her support and A. Kros for the helpful discussions. AFM measurements were performed in the AFM Lab of the Leiden Institute of Physics.

\section{REFERENCES}

(1) Fu, W.; Jiang, L.; van Geest, E. P.; Lima, L. M. C.; Schneider, G. Sensing at the Surface of Graphene Field-Effect Transistors. Adv. Mater. 2017, 29, 1603610.

(2) Arjmandi-Tash, H.; Belyaeva, L. A.; Schneider, G. Single molecule detection with graphene and other two-dimensional materials: nanopores and beyond. Chem. Soc. Rev. 2016, 45 (3), 476-493.

(3) Gobbi, M.; Orgiu, E.; Samori, P. When 2D Materials Meet Molecules: Opportunities and Challenges of Hybrid Organic/ Inorganic van der Waals Heterostructures. Adv. Mater. 2018, 1706103.

(4) Tabaei, S. R.; Ng, W. B.; Cho, S.-J.; Cho, N.-J. Controlling the Formation of Phospholipid Monolayer, Bilayer, and Intact Vesicle Layer on Graphene. ACS Appl. Mater. Interfaces 2016, 8, 1187511880 .

(5) Willems, N.; Urtizberea, A.; Verre, A. F.; Iliut, M.; Lelimousin, M.; Hirtz, M.; Vijayaraghavan, A.; Sansom, M. S. P. Biomimetic Phospholipid Membrane Organization on Graphene and Graphene Oxide Surfaces: A Molecular Dynamics Simulation Study. ACS Nano 2017, 11, 1613-1625.

(6) Gao, L.; Ni, G.-X.; Liu, Y.; Liu, B.; Neto, A. H. C.; Loh, K. P. Face-to-Face Transfer of Wafer-Scale Graphene films. Nature 2014, 505, 190-194.

(7) Wood, J. D.; Doidge, G. P.; Carrion, E. A.; Koepke, J. C.; Kaitz, J. A.; Datye, I.; Behnam, A.; Hewaparakrama, J.; Aruin, B.; Chen, Y.; Dong, H.; Haasch, R. T.; Lyding, J. W.; Pop, E. Annealing Free, Clean Graphene Transfer Using Alternative Polymer Scaffolds. Nanotechnology 2015, 26, 055302.

(8) Arjmandi-Tash, H.; Allain, A.; Han, Z.; Bouchiat, V. Large Scale Integration of CVD-Graphene Based NEMS with Narrow Distribution of Resonance Parameters. 2D Materials 2017, 4, 025023.

(9) Belyaeva, L. A.; Fu, W.; Arjmandi-Tash, H.; Schneider, G. F. Molecular Caging of Graphene with Cyclohexane: Transfer and Electrical Transport. ACS Cent. Sci. 2016, 2, 904-909.

(10) (a) Pumera, M.; Ambrosi, A.; Bonanni, A.; Chng, E. L. K.; Poh, H. L. Graphene for Electrochemical Sensing and Biosensing. TrAC, Trends Anal. Chem. 2010, 29 (9), 954-965. (b) Arjmandi-Tash, H.; Lebedev, N.; van Deursen, P.; Aarts, J.; Schneider, G. F. Hybrid cold and hot-wall chamber for fast synthesis of uniform graphene. Carbon 2017, 118, 438-442.

(11) Li, X.; Cai, W.; An, J.; Kim, S.; Nah, J.; Yang, D.; Piner, R.; Velamakanni, A.; Jung, I.; Tutuc, E.; Banerjee, S. K.; Colombo, L.; Ruoff, R. S. Large-Area Synthesis of High-Quality and Uniform Graphene Films on Copper Foils. Science 2009, 324, 1312-1314.

(12) Suk, J. W.; Kitt, A.; Magnuson, C. W.; Hao, Y.; Ahmed, S.; An, J.; Swan, A. K.; Goldberg, B. B.; Ruoff, R. S. Transfer of CVD-Grown Monolayer Graphene onto Arbitrary Substrates. ACS Nano 2011, 5, 6916-6924.

(13) Chen, Y.; Gong, X.-L.; Gai, J.-G. Progress and Challenges in Transfer of Large-Area Graphene Films. Adv. Sci. 2016, 3, 1500343.

(14) Kaganer, V. M.; Möhwald, H.; Dutta, P. Structure and phase transitions in Langmuir monolayers. Rev. Mod. Phys. 1999, 71, 779.

(15) Li, W.; Chung, J. K.; Lee, Y. K.; Groves, J. T. GrapheneTemplated Supported Lipid Bilayer Nanochannels. Nano Lett. 2016, $16,5022-5026$.

(16) Lin, W.-H.; Chen, T.-H.; Chang, J.-K.; Taur, J.-I.; Lo, Y.-Y.; Lee, W.-L.; Chang, C.-S.; Su, W.-B.; Wu, C.-I. A Direct and Polymer-Free Method for Transferring Graphene Grown by Chemical Vapor Deposition to Any Substrate. ACS Nano 2014, 8, 1784-1791.
(17) Regan, W.; Alem, N.; Alemán, B.; Geng, B. S.; Girit, C.; Maserati, L.; Wang, F.; Crommie, M.; Zettl, A. A direct transfer of layer-area graphene. Appl. Phys. Lett. 2010, 96, 113102.

(18) Zhang, G.; Güell, A. G.; Kirkman, P. M.; Lazenby, R. A.; Miller, T. S.; Unwin, P. R. Versatile Polymer-Free Graphene Transfer Method and Applications. ACS Appl. Mater. Interfaces 2016, 8, 8008-8016.

(19) Girard-Egrot, A. P.; Blum, L. J. Nanobiotechnology of Biomimetic Membranes; Springer, 2007; Vol. 1.

(20) Klopfer, K. J.; Vanderlick, T. K. Isotherms of Dipalmitoylphosphatidylcholine (DPPC) Monolayers: Features Revealed and Features Obscured. J. Colloid Interface Sci. 1996, 182, 220-229.

(21) Baoukina, S.; Monticelli, L.; Marrink, S. J.; Tieleman, D. P. Pressure-Area Isotherm of a Lipid Monolayer from Molecular Dynamics Simulations. Langmuir 2007, 23, 12617-12623.

(22) Huang, P. Y.; Ruiz-Vargas, C. S.; van der Zande, A. M.; Whitney, W. S.; Levendorf, M. P.; Kevek, J. W.; Garg, S.; Alden, J. S.; Hustedt, C. J.; Zhu, Y.; Park, J.; McEuen, P. L.; Muller, D. A. Grains and Grain Boundaries in Single-Layer Graphene Atomic Patchwork Quilts. Nature 2011, 469, 389-392.

(23) Li, X.; Zhu, Y.; Cai, W.; Borysiak, M.; Han, B.; Chen, D.; Piner, R. D.; Colombo, L.; Ruoff, R. S. Transfer of Large-Area Graphene Films for High-Performance Transparent Conductive Electrodes. Nano Lett. 2009, 9, 4359-4363.

(24) Su, Y.; Han, H.-L.; Cai, Q.; Wu, Q.; Xie, M.; Chen, D.; Geng, B.; Zhang, Y.; Wang, F.; Shen, Y. R; Tian, C. Polymer Adsorption on Graphite and CVD Graphene Surfaces Studied by Surface-Specific Vibrational Spectroscopy. Nano Lett. 2015, 15, 6501-6505.

(25) Pirkle, A.; Chan, J.; Venugopal, A.; Hinojos, D.; Magnuson, C. W. The Effect of Chemical Residues on the Physical and Electrical Properties of Chemical Vapor Deposited Graphene Transferred to $\mathrm{SiO}_{2}$. Appl. Phys. Lett. 2011, 99, 122108.

(26) Kumar, K.; Kim, Y.-S.; Yang, E.-H. The Influence of Thermal Annealing to Remove Polymeric Residue on the Electronic Doping and Morphological Characteristics of Graphene. Carbon 2013, 65, 3545 .

(27) Meléndrez, D.; Jowitt, T.; Iliut, M.; Verre, A. F.; Goodwin, S.; Vijayaraghavan, A. Adsorption and binding dynamics of graphenesupported phospholipid membranes using the QCM-D technique. Nanoscale 2018, 10, 2555-2567.

(28) Hirtz, M.; Oikonomou, A.; Clark, N.; Kim, Y.-J.; Fuchs, H.; Vijayaraghavan, A. Self-limiting multiplexed assembly of lipid membranes on large-area graphene sensor arrays. Nanoscale 2016, 8, 15147-15151.

(29) Baoukina, S.; Monticelli, L.; Risselada, H. J.; Marrink, S. J.; Tieleman, D. P. The molecular mechanism of lipid monolayer collapse. Proc. Natl. Acad. Sci. U.S.A. 2008, 105, 10803-10808.

(30) Arjmandi-Tash, H.; Jiang, L.; Schneider, G. F. Rupture index: A quantitative measure of sub-micrometer cracks in graphene. Carbon 2017, 118, 556-560.

(31) Lima, L. M. C.; Fu, W.; Jiang, L.; Kros, A.; Schneider, G. F. Graphene-stabilized lipid monolayer heterostructures: a novel biomembrane superstructure. Nanoscale 2016, 8, 18646-18653. 